\title{
Combining Local and Global Hypotheses in Deep Neural Network for Multi-label Image Classification
}

\author{
Qinghua Yu ${ }^{\mathrm{a}}$, Jinjun Wang ${ }^{\mathrm{a}, *}$, Shizhou Zhang ${ }^{\mathrm{a}}$, Yihong Gong ${ }^{\mathrm{a}}$, Jizhong Zhao ${ }^{\mathrm{b}}$ \\ ${ }^{a}$ Institute of Artificial Intelligence and Robotic, Xi'an Jiaotong University, \\ Xian Ning West Road No.28, Shaanxi, P.R. China, 710049 \\ ${ }^{b}$ School of Electronic \& Information Engineering, Xi'an Jiaotong University
}

\begin{abstract}
Multi-label image classification is a challenging problem in computer vision. Motivated by the recent development in image classification performance using Deep Neural Networks, in this work, we propose a flexible deep Convolutional Neural Network (CNN) framework, called Local-Global-CNN (LGC), to improve multi-label image classification performance. LGC consists of firstly a local level multi-label classifier which takes object segment hypotheses as inputs to a local CNN. The output results of these local hypotheses are aggregated together with max-pooling and then re-weighted to consider the label co-occurrence or interdependencies information by using a graphical model in the label space. LGC also utilizes a global CNN that is trained by multilabel images to directly predict the multiple labels from the input. The predictions of local and global level classifiers are finally fused together to obtain MAP estimation of the final multi-label prediction. The above LGC framework could benefit from a pre-train process with a large-scale single-label image dataset, e.g., ImageNet. Experimental results have shown that the proposed framework could achieves promising performance on Pascal VOC2007 and VOC2012 multi-label image dataset.
\end{abstract}

Keywords: Deep Learning, Convolutional Neural Network, Multi-label Classification

${ }^{*}$ Corresponding author

Preprint submitted to Journal of $\mathrm{BT}_{E} X$ Templates

September 25, 2016

(C) 2016. This manuscript version is made available under the Elsevier user license http://www.elsevier.com/open-access/userlicense/1.0/ 


\section{Introduction}

With the development of Internet and digital camera equipment, the amount of digital images is drastically increasing in recent years. Image annotation is an important task to automate the processing of such huge image data. Image annotation requires identifying a set of objects presented in each image from a given set of desired object concepts, and usually such prediction process is relevant to multi-label image classification. Many existing image classification works [1,2,3] focus on single-label problems, with the most recent advances being reported on the large-scale ImageNet database [1]. Compared with designing handcrafted visual features, e.g., SIFT [4],

10 Fisher Vector [5] and combining with classic classifier such as Support Vector Machine (SVM) [6], deep Convolutional Neural Network (CNN) has demonstrated promising results for single-label image classification tasks due to its ability to discover representative and abstract features automatically [7].

Multi-label image classification task is more complicated than single-label image classification due to the following challenges. Firstly, foreground objects in multilabel images are not as aligned as in single-label images. In some multi-label images, objects are located at various positions with different scales and poses. Even worse, some foreground objects are occluded by others. For example, in a living room scene, the chairs are located at various positions, and may be occluded by tables. Secondly, in most single-label images, a foreground object occupy a larger portion of entire image, and the remaining local regions are often clean and semantically very different from the object. However, there are more than one objects in most of multi-label images, and the foreground object may only occupy a very small local region of the entire image.

Despite the challenges, there are also additional cues that could be utilized to assist multi-label image classification. Co-occurrence and interdependencies exist among multiple class labels, and capturing the co-occurrence information among class labels is useful to improve performance. For example, chair and dining table usually appear together, while the former is unlikely to co-appearance with sheep. Typically, many parts of the chairs could be invisible, but the dining table is easy to recognize and 
almost impossible to appear together with dining table could be lower ranked. Besides, advantages of deep CNN are also beneficial for multi-label image classification. For instance, Gong et al. [8] compared several conventional visual features, such as SIFT [4] and HOG [9], with features based on deep neural networks for the multi-label annotation problem, and it is observed that the latter outperforms the former.

In this paper, we are most interested at combining the local and global hypotheses to improve multi-label image classification performance. Local region pays attention to a single object, while global multi-label image emphasizes the global level information. Local regions and global multi-label image consist of different information from different perspective and are both important for multi-label classification task. Motivated by these observations, in this paper, we propose a flexible deep CNN framework for multi-label image classifier, called Local-Global-CNN (LGC). LGC consists of firstly a local level multi-label classifier which takes object segment hypotheses as inputs to a local CNN model, and the output results of different hypotheses are then aggregated with max-pooling and then re-weighted to consider the label co-occurrence information by using a graphical model in the label space. The local CNN model is indeed a single-label classifier to predict over a set of local object segment hypotheses [10], and the goal of using a graph to refine the local prediction is to find the relevant (co-occurred) and irrelevant (uncorrelated) labels, because most of irrelevant labels we found are true negative labels in the multi-label image, and therefore we could significantly reduce prediction from irrelevant labels and reduce the effects of those noise hypotheses. LGC also consists of a second CNN model as the global level multi-label classifier, where images are directly fed into the CNN and generate multi-label prediction. Finally, we integrate predictions of local and global level classifier together as the ultimate multi-label prediction. In this way, the local level classifier and the global level classifier compensate the shortcomings of each other. The proposed LGC framework nicely integrates these two aspects by taking advantage of local level, global level and label co-occurrence information and achieves very promising performance on Pascal VOC 2007 and VOC 2012 [11] multi-label image dataset. The rest of the paper is organized as follows. In Section 2, we briefly review the related work. Section 3 and section 4 present details of local level multi-label classification and global level 


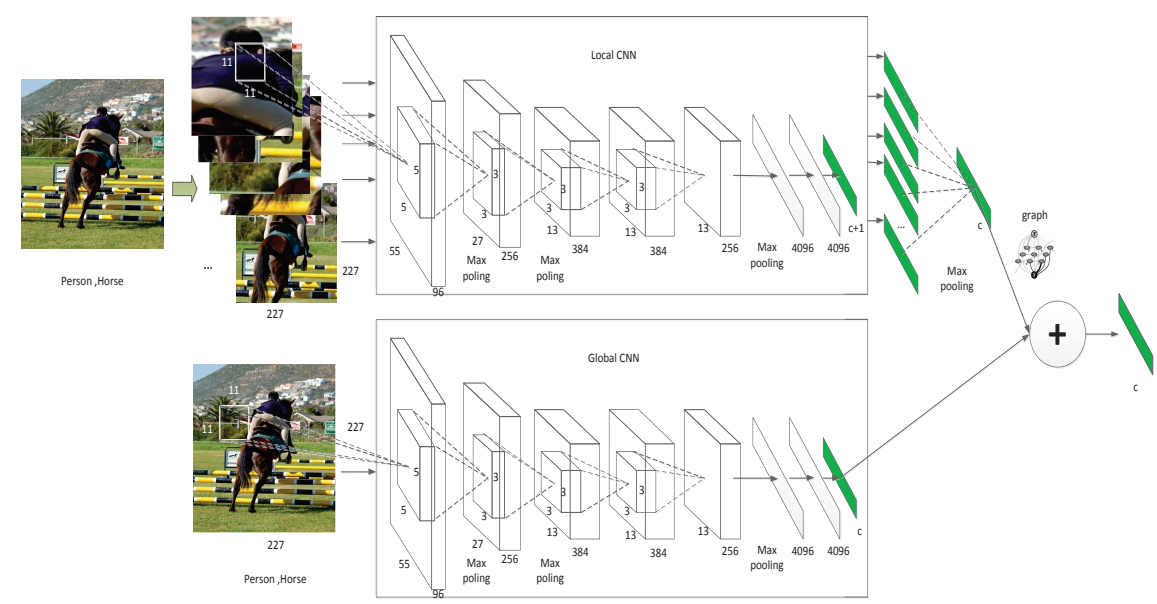

Figure 1: An illustration of the proposed LGC framework

multi-label classification respectively, and Section 5 combines the two and presents the complete LGN framework. Finally, experimental results and conclusions are provided in section 6 and section 7 respectively.

\section{Related Work}

A considerable amount of research has been devoted to multi-label classification problems. Over the past decade, many traditional methods are based on bag-of-words (BoW). Some complex methods exploit label correlations to improve multi-label image classification performance. In recent years, Deep Neural Networks have demonstrated promising performance in image classification. In this section, we will provide a brief review over the most related work to multi-label classification.

The Bag-of-features (BoF) model as a traditional approach has been very popular in image classification. The BoF method represents an image as a histogram of its local features. Hand-crafted features, such as SIFT [4], Histogram of Oriented Gradients [12] and Local Binary Patterns [13] are firstly extracted from local patches, quantized into discrete visual words, and then converted into a compact histogram as the image representations. Taking information about the spatial layout of features into consideration, the SPM [14] method partitions the image into increasingly finer spa- 
tial subregions and computes histograms of local features from each sub-region. The resulted spatial pyramid is a computationally efficient extension of the orderless BoF representation, and has shown very promising performance on many image classification tasks. Some coding schemes, e.g., Sparse Coding [15] and Locality-constrained Linear Coding (LLC) [16], replace the VQ coding in traditional SPM to further improve performance in image classification.In addition, some methods $[17,18,19]$ focus on image data representation by subspace learning and feature selection. The method in [17] propose a novel Robust Structured Subspace Learning (RSSL) algorithm by integrating image understanding and feature learning into a joint learning framework. The learned subspace is adopted as an intermediate space to reduce the semantic gap between the low-level visual features and the high-level semantics.

In contrast to traditional methods that focus on feature representation, the labels co-occurrence information is very important in multi-label classification. Many works explore correlation between labels to improve multi-label classification performance. One group of works is the ranking based methods [20, 21, 22], where the relevant label$\mathrm{s}$ are ranked higher than irrelevant ones in considering the label correlations. Another group of works is the graph-based methods that incorporate the label correlations into label propagation algorithms as part of the graph weights [23, 24]. Others have used the label correlations as additional constraints [25, 26]. The method in [27] explore label combinations to enrich the label space and improve the label identification capacity in the original label space by generating many label combination pairs in a tree-structured graph for the label space, according to the label co-occurrence information in the training data, which naturally forms a conditional random field.

Recently, features based on deep neural networks have demonstrated promising performance in image classification. Deep Neural Networks has achieved promising performance in many computer vision tasks. Specifically, deep CNN has achieved state-of-the-art performance $[28,29,7,30]$ in many single-label dataset, such as CIFAR10/100 [31] and ImageNet [1]. Many researchers have therefore adopted the CNN architectures to address multi-label classification problems. Gong et al. [8] use a similar network structure to [7] for the multi-label annotation problem. He compared several multi-label loss functions, and found that the weighted approximated-ranking loss 
Yun et al. [33] also proposed a novel CNN structure, called Hypotheses-CNN-Pooling (HCP). Object segment hypotheses are taken as the inputs. Output results of these hypotheses from a shared $\mathrm{CNN}$ are aggregated with max pooling to produce the ultimate multi-label predictions. The proposed HCP infrastructure has achieved promising performance on Pascal VOC2007 and VOC2012 multi-label image dataset. Some studies $[34,35,36]$ have shown that $\mathrm{CNN}$ features are suitable for images classifications.

\section{Local Level Multi-label Classification}

Local level multi-label classifier takes object segment hypotheses as inputs, and aggregate the output results of different hypotheses with max-pooling. The aggregated result will be re-weighted to consider the label co-occurrence information by using a graphical model in the label space. In this section, we will introduce training stage and test stage of local level multi-label classification.

\subsection{Training Stage}

Local CNN is a single-label classifier. The architecture of the local CNN is similar to the network described in [7], which contains five convolutional layers and three fully-connected layers with about 60 million parameters. To obtain an effective CNN model, we need enough training dataset. Local CNN training dataset is provided by ground-truth bounding box information of multi-label images training dataset. We crop out the ground-truth bounding box as the training dataset. However, large-scale multi-label dataset is not affordable and difficult to annotate. What's more, we also need the object's bounding box ground-truth and corresponding label ground-truth in multi-label dataset. Bounding box and label annotation will cost a lot of manpower and resources. Fortunately, the CNN can be pre-trained by a large-scale single-label image dataset for parameter initialization. To obtain an optimal CNN model for our task,fine-tuning is enforced to adjust the parameters. 


\subsubsection{Pre-training On A Large-scale Single-label Image Dataset}

We pre-train the local CNN on ImageNet [1]. Before the start of training, we first resize images to $256 \times 256$. Next, random $227 \times 227$ patches from the image are fed to convolutional layers. We set convolution filter sizes to squares of size 11, 5, and 3 respectively for the different convolutional layers. Max pooling layers are used in some of convolutional layers. Each of the densely connected layers are followed by a dropout layers with a dropout ratio of 0.6. The output of the last fully-connected layer is fed to a 1000-way softmax layer with multinomial logistic regression as the loss function. The rectified linear units (ReLU) is chose as the nonlinear activation function for all layers. The whole network is optimized by stochastic gradient descent with a momentum of 0.9 and weight decay of 0.0005 . The global learning rate for the all layers is set to 0.01 at the beginning, and a staircase weight decay is applied after a few epochs.

\subsubsection{Fine-tuning}

Fine-tuning process is enforced to adapt the model for our classification task. Firstly, we need prepare the single-label images from a multi-label dataset, e.g., Pascal VOC [11]. And object boundingbox annotations are required for multi-label image dataset. We generate some windows around the ground-truth boundingbox and select several windows where overlapping rate between the ground-truth boundingbox and the window is more than $70 \%$. We crop out the selected window as the training image and annotate it with the same label with ground-truth boundingbox. In addition, we add a negative class. The negative class images are selected from windows whose overlapping rate is less than $10 \%$. Each training image is resized into $230 \times 230$. Random $227 \times 227$ patches extracted from the given image are fed to network. Fine-tuning process is different from pre-training in some details. Since the category number of Pascal VOC is not equal to ImageNet, the output of the last fully-connected layer is fed into a $(c+1)$-way softmax which produces a probability distribution over the $(c+1)$ class labels. $c$ is category number of Pascal VOC and 1 refers to the negative class. The loss function is still multinomial logistic regression. During fine-tuning process, 
rameters pre-trained on ImageNet. The parameters of the last fully-connected layer are initialed with a Gaussian distribution $G(\mu, \sigma)(\mu=0, \sigma=0.01)$. We set much higher learning rate for the last fully-connected layer to guarantee a fast convergence to the new optimum on the new target dataset. time-consuming when extract a large number of hypotheses.

Most recently, Cheng et al. [10] proposed a simple and efficient hypotheses extraction approach called binarized normed gradients(BING). BING can be used for efficient objectness estimation, which requires only a few atomic operations. This method and yielding $96.2 \%$ object detection rate (DR) with 1,000 proposals. In the experiment, we choose BING as our hypotheses extraction method. 


\subsubsection{Max Pooling}

$n$ hypotheses of a multi-label image are fed into local CNN and $n(c+1)$-dimensional vectors are computed as the output of the CNN. Some hypotheses may contain one object or more. Others may contain none of object. To suppress the noisy hypotheses , we fuse the outputs into one integrative prediction with a cross-hypothesis max-pooling method, and remove negative class output. As for hypothesis which contains an object, the output vector will have a high response on the $j_{t h}$ component, meaning a high confidence for the appearance of $j_{t h}$ category in multi-label image. As for hypothesis which contains none object, the output vector will have a high response on the negative class component and have low response on the remaining class component. In this way, we suppress the influence of noisy hypotheses. Suppose $\mathbf{v}_{i}(i=1, \ldots, n)$ is the output vector of the $i_{t h}$ hypothesis from the local CNN and $\mathbf{v}_{i}^{j}$ is the $j_{t h}$ component of $\mathbf{v}_{i}$. And we remove negative class component. The cross-hypothesis max-pooling on the $n$ output vectors can be formulated as,

$$
\mathbf{v}^{j}=\max \left(\mathbf{v}_{1}^{j}, \mathbf{v}_{2}^{j}, \ldots, \mathbf{v}_{n}^{j}\right)
$$

where $\mathbf{v}^{j}$ can be considered as the predicted value for the $j_{t h}$ category of the given image. With cross-hypothesis max-pooling, large predicted values corresponding to objects of interest will be reserved, while the values from the noisy hypotheses will be ignored.

\subsubsection{Label Co-occurrence Information}

Capturing the dependencies among class labels during classification is useful to improve classification performance. We construct a simple graph to find the related labels and irrelated labels of the given multi-label image. A fully connected directed graph $G=(V, E)$ is constructed over the label space. Each vertice of $V$ represents a label variable, and a set of directed edges $(s, t) \in E$ represents the dependency between the label variables. We measure co-presence empirical probabilities of each label pair as the weight of the corresponding edge. We propose a simple new measure to compute co-presence empirical conditional probabilities from the training data. For 
two label variables $V_{i}$ and $V_{j}$, the weight of the directed edge $W\left(\overrightarrow{V_{i}, V_{j}}\right)$ is defined as,

$$
W\left(\overrightarrow{V_{i}, V_{j}}\right)=\frac{\operatorname{count}\left(V_{i}, V_{j}\right)}{\operatorname{count}\left(V_{i}\right)}
$$

where $\operatorname{count}\left(V_{i}, V_{j}\right)$ is the number of co-occurrence of the two labels in the training data, such that,

$$
\operatorname{count}\left(V_{i}, V_{j}\right)=\sum_{\ell=0}^{n} I\left[V_{i}^{\ell}=1, V_{j}^{\ell}=1\right]
$$

and $I[\bullet]$ denotes an indicator function. Similarly, $\operatorname{count}\left(V_{i}\right)$ and $\operatorname{count}\left(V_{j}\right)$ are the numbers of occurrences of single labels in the training data.

For example, there are totally 100 people images, 80 dog images and 15 images containing people and dogs. Assume label variables $V_{i}$ and $V_{j}$ represent person and dog respectively. The weight of the directed edge $W\left(\overrightarrow{V_{i}, V_{j}}\right)$ is $15 / 100$, and $W\left(\vec{V}_{j}, V_{i}\right)$ is $15 / 80$.

Our task is to find the related labels and irrelated labels of the given multi-label images. This problems can be formulated in terms of energy minimization. The energy function is defined as,

$$
E=\sum_{i=1}^{c}\left[\alpha\left(L_{i}-S_{i}\right)^{2}+\sum_{j=1}^{c}\left(L_{i}-L_{j}\right)^{2} * W\left(\vec{V}_{i}, V_{j}\right)\right]
$$

where $L_{i}$ represents whether the $i_{t h}$ category is the related label of multi-label image or not. At the beginning, $L_{i}$ will be random assigned a label 1 or 0 , which represent related label and irrelated label respectively. $S_{i}$ is the prediction value of the multi-label image on the $i_{t h}$ category. $\left(L_{i}-S_{i}\right)^{2}$ is the data item which measures the disagreement between $L$ and the observed data $S . \sum_{j=1}^{c}\left(L_{i}-L_{j}\right)^{2} * W\left(\vec{V}_{i}, V_{j}\right)$ is the smooth data, which measures the extent of piecewise smooth. $\alpha$ is a ratio adjustment factor. Some methods (e.g. graph cut and simulated annealing), can find a local minimum of the energy minimization. From the result of $L$, we get the related labels and irrelated labels of the given multi-label images.

According to the above results, the amount of the related labels we found is usually more than the ground-truth. But most of the irrelated labels we found are true negative labels of the multi-label image. We reduce prediction values of irrelated labels by half to reduce impact of noise hypotheses and remain unchanged for related labels. In the way, we further improve the accuracy of multi-label classification. 


\section{Global Level Multi-label Classification}

ilar to the network described in [7], which contains five convolutional layers and three fully-connected layers with about 60 million parameters. Therefore, without enough training images, it is very difficult to obtain an effective CNN model for multi-label classification. The same as the local CNN, we use the a large-scale single-label image for our task, the entire images from a multi-label image set, e.g., Pascal VOC [11], are then utilized to fine-tune the $\mathrm{CNN}$ model.

In the fine-tuning process, parameters pre-trained on ImageNet are directly transferred for fine-tuning except for the last fully-connected layer. Each image is resized into $227 \times 227$ pixels without cropping. Since the category number of Pascal VOC is not equal to that of ImageNet, the output of the last fully-connected layer is fed into a c-way softmax which produces a probability distribution over the $c$ class labels. The loss function is softmax loss. The softmax loss function is defined as follows.

Assuming that each image has multiple labels, and we can form a label vector $\mathbf{y} \in R^{1 \times c}$ where $\mathbf{y}_{j}=1$ means the presence of a label and $\mathbf{y}_{j}=0$ means absence of a label for an image. We can obtain ground-truth probability by normalizing $\mathbf{y}$ as $\mathbf{y} /\|\mathbf{y}\|^{2}$. If the ground-truth and prediction probability for image $i$ and class $j$ is defined as $\bar{p}_{i j}$ and $p_{i j}$ respectively, the cost function to be minimized is,

$$
J=-\frac{1}{m} \sum_{i=1}^{n} \sum_{j=1}^{c} \bar{p}_{i j} \log \left(p_{i j}\right)=-\frac{1}{m} \sum_{i=1}^{n} \sum_{j \in \mathcal{L}_{i}} \frac{1}{c_{+}} \log \left(p_{i j}\right)
$$

where $\mathcal{L}_{i}$ denotes the positive label set of the $i^{\text {th }}$ image and $c_{+}$denotes the number of positive labels for each image.

we also adopt a discriminating learning rate scheme for different layers. The parameters of the last fully-connected layer are initialed with a Gaussian distribution $G(\mu, \sigma)(\mu=0, \sigma=0.01)$. We set a much higher learning rate for the last fullyconnected layer to guarantee a fast convergence to the new optimum on the new target 


\section{Combination of Local and Global Level Multi-label Classification}

Finally, we add predictions of local level multi-label classification and global level multi-label classification together as the ultimate multi-label predictions. From different perspective, local level multi-label classification and global level multi-label classification make up for the shortcomings of each other. Our deep CNN infrastructure nicely integrates these two aspects by taking advantage of local level, global level and label co-occurrence information and achieves very promising performance on Pascal VOC2007 and VOC2012 multi-label image dataset.

Based on the trained LGC model, the multi-label classification of a given image can be summarized as follows. We firstly generate the input hypotheses of the given image based on BING [10] method. The object segment hypotheses are taken as inputs to local CNN. Predictions of different hypotheses are aggregated with max pooling. Then we take the label co-occurrence or interdependencies information into account to improve classification accuracy by using graphs in the label space. As for global level multi-label classifier, the global multi-label images are taken as inputs to global CNN. Finally, we add predictions of local level multi-label classifier and global level multi-label classifier together as the ultimate multi-label prediction. As shown in Fig 2, the left part is local level classification. For each object independent hypothesis, there is a high response on the corresponding category (i.e.,cow, person) after classification of local CNN. Some categories (e.g.,horse ,sheep ) are similar to cow and also have high response. Taking the co-occurrence information into account, we find the irrelated labels (e.g., cat) and reduce their prediction values by half. The right part is global level classification. The entire image is taken as input to global CNN. The response is high on several categories. Finally, we add predictions of local level multi-label classifier and global level multi-label classifier together as the ultimate multi-label prediction.

\section{Experimental Results}

\subsection{Dataset}

We performed experiments on the PASCAL Visual Object Classes Challenge (VOC) dataset [11]. Pascal is one of the most well-known image datasets for classification and 


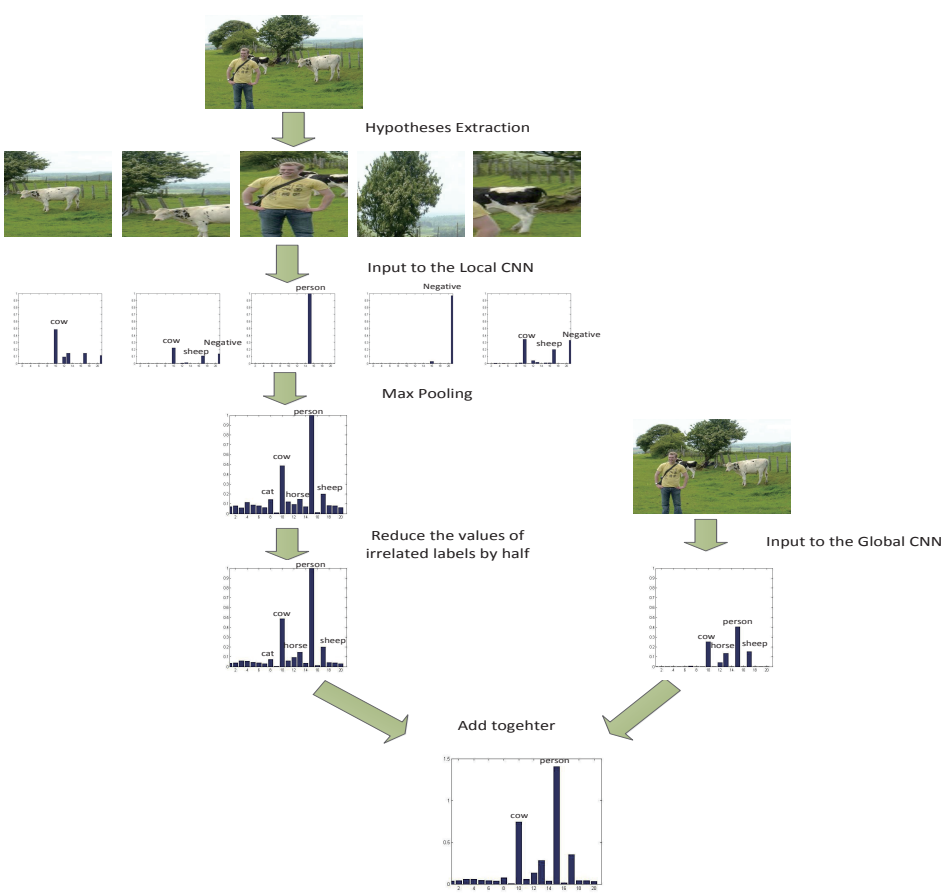

Figure 2: An illustration of the proposed LGC for a VOC 2007 test image. The left part is local level classification. Firstly, object independent hypothesis are taken as inputs to local CNN. Secondly,taking the co-occurrence information into account, we reduce irrelated labels prediction values by half. The right part is global level classification. The entire image is taken as input to global CNN. Finally, we add predictions of local level multi-label classifier and global level multi-label classifier together as the ultimate multi-label prediction. 
detection. It contains 20 different object classes and is widely used as a benchmark for multi-label classification. In this paper, we conducted experiments on PASCAL VOC 2007 and VOC 2012 dataset. These two dataset, which contain 9,963 and 22,531 images respectively, are divided into training, validation and testing subsets. We followed the suggested data partition and evaluation metric which is the Average Precision (AP) measure and mean AP (mAP) to comply with the PASCAL challenge protocols.

\subsection{Benchmark Methods}

We compare our method with several state-of-the-art approaches as listed below. The deep learning methods are all pre-trained on the original ImageNet dataset [1] with 1000 categories and the network is similar to [7]. In addition, we also compared with global level classification (Global-Cls) and local level classification (Local-Cls). To validate the effectiveness of graph model in local level classification, we present the result of local level classification without using graph model. In other words, we only use the combination of local CNN and cross-hypothesis max-poling, which is named as Local-CNN-Max.

- INRIA [41]: Harzallah et al. proposed a contextual combination method of localization and classification to improve the performance for both. Specifically, for classification, image representation is built on the traditional feature extractioncoding-pooling pipeline, and object localization is built on sliding-widow approaches. Furthermore, the localization is employed to enhance the classification performance.

- FV [5]: The Fisher Vector representation of images can be considered as an extension of the bag-of-words. Some well-motivated strategies, e.g., $L 2$ normalization, power normalization and spatial pyramids, are adopted over the original Fisher Vector to boost the classification accuracy.

- CNN-SVM [36]: OverFeat [42], which obtained very competitive performance in the image classification task of ILSVRC 2013, was released by Sermanet et al. as a feature extractor. Razavian et al. [36] employed OverFeat, which is pre-trained on ImageNet, to get CNN activations as the off-the-shelf features. 
The state-of-the-art classification result on PASCAL VOC 2007 was achieved by 1000C [33] on 9 categories AP. These results demonstrated the effectiveness of the 
Table 1: Comparison of classification results (AP in \%) on VOC 2007 (trainval/test). * indicates methods using external data (e.g. ImageNet) for training.

\begin{tabular}{|c|c|c|c|c|c|c|c|c|c|c|c|c|c|c|c|c|c|c|c|c|}
\hline & plane bike & bird & boat & bottle & e bus & car & cat & chair & cow & table & $\operatorname{dog}$ & horse & motor & r person & inplant & $t$ sheep s & sofa & train & tv & mAP \\
\hline INRI & $7.2 \quad 69.3$ & 56.2 & 66.6 & 45.5 & 68.1 & 83.4 & 53.6 & 58.3 & 51.1 & 62.2 & 45.2 & 78.4 & 69.7 & 86.1 & 52.4 & 54.45 & 54.3 & 75.8 & 62.1 & 63.5 \\
\hline $\mathrm{FV}[5]$ & $5.7 \quad 64.8$ & 52.8 & 70.6 & 30.0 & 64.1 & 77.5 & 55.5 & 55.6 & 41.8 & 56.3 & 41.7 & 76.3 & 64.4 & 82.7 & 28.3 & 39.7 & 56.6 & 79.7 & 51.5 & 58.3 \\
\hline CNN-S & 8.581 .0 & 83.5 & 82.0 & 42.0 & 72.5 & 85.3 & 81.6 & 59.9 & 58.5 & 66.5 & 77.8 & 81.8 & 78.8 & 90.2 & 54.8 & 71.1 & 62.6 & 87.4 & 71.8 & 73.9 \\
\hline PRE-1C & $8.5 \quad 81.5$ & 87.9 & 82.0 & 47.5 & 75.5 & 90.1 & 87.2 & 61.6 & 75.7 & 67.3 & 85.5 & 83.5 & 80.0 & 95.6 & 60.8 & 76.8 & 58.0 & 90.4 & 77.9 & 77.7 \\
\hline HCP- & $\begin{array}{ll}1 & 90.1\end{array}$ & 92.8 & 89.9 & 51.5 & 80.0 & 91.7 & 91.6 & 57.7 & 77.8 & 70.9 & 89.3 & 89.3 & 85.2 & 93.0 & 64.0 & 85.7 & 62.7 & 94.4 & 78.3 & 81.5 \\
\hline Globa & $1.6 \quad 84.9$ & 89.2 & 84.9 & 41.9 & 75.1 & 88.8 & 86.1 & 60.6 & 66.0 & 74.4 & 83.1 & 86.3 & 83.2 & 91.6 & 58.6 & 74.7 & 59.4 & 91.5 & 73.1 & 77.3 \\
\hline Local-CNN- & $86.3 \quad 84.7$ & 83.9 & 77.0 & 57.2 & 77.8 & 91.7 & 84.5 & 54.7 & 63.6 & 65.4 & 84.6 & 84.7 & 86.7 & 96.5 & 68.5 & 71.4 & 64.6 & 89.7 & 70.7 & 77.2 \\
\hline Local-Cls" & $\begin{array}{lll}90.8 & 87.7\end{array}$ & 87.3 & 80.3 & 57.9 & 79.9 & 92.7 & 87.7 & 58.3 & 68.3 & 66.9 & 86.6 & 85.3 & 88.6 & 95.8 & 73.0 & 76.0 & 65.1 & 92.0 & 74.7 & 79.8 \\
\hline LGC* & $92.8 \quad \mathbf{9 0 . 8}$ & 90.8 & 85.3 & 56.3 & 81.4 & 93.1 & 89.5 & 65.3 & 71.5 & 76.3 & 88.1 & 88.6 & 89.7 & 96.0 & 70.6 & 78.8 & 68.9 & 93.9 & 77.8 & 82.3 \\
\hline
\end{tabular}

Table 2: Comparison of classification results (AP in \%) on VOC 2012 (trainval/test). * indicates methods using external data (e.g. ImageNet) for training.

\begin{tabular}{|c|c|c|c|c|c|c|c|c|c|c|c|c|c|c|c|c|c|c|}
\hline & planebike bird boat & bottle & e bus & car & cat & chair & cow & table & $\operatorname{dog}$ & horse & motor & or persol & onplant & t sheep & p sofa & train & $\mathrm{tv}$ & $\mathrm{mAP}$ \\
\hline LeCun-ICML* [44] & 96.077 .188 .485 .5 & 55.8 & 85.8 & 78.6 & 91.2 & 65.0 & 74.4 & 67.7 & 87.8 & 86.0 & 85.1 & 90.9 & 52.2 & 83.6 & 61.1 & 91.8 & 76.1 & 79.0 \\
\hline PRE-1000C* [43] & 93.578 .487 .780 .9 & 57.3 & 85.0 & 81.6 & 89.4 & 66.9 & 73.8 & 62.0 & 89.5 & 83.2 & 87.6 & 95.8 & 61.4 & 79.0 & 54.3 & 88.0 & 78.3 & 78.7 \\
\hline HCP-1000* [33] & $\mathbf{9 7 . 7} 83.093 .2 \quad 87.2$ & 59.6 & 88.2 & 81.9 & 94.7 & 66.9 & 81.6 & 68.0 & 93.0 & 88.2 & 87.7 & 92.7 & 59.0 & 85.1 & 55.4 & 93.0 & 77.2 & 81.7 \\
\hline LGC* & $5.8 \mathbf{8 3 . 3} 89.6 \mathbf{8 7 . 2}$ & 63.4 & 89.3 & 84.4 & 91.6 & 69.5 & 74.9 & 67.6 & 91.1 & 85.3 & 89.3 & 94.6 & 64.6 & 79.4 & 61.1 & 90.8 & 76.7 & 81.5 \\
\hline
\end{tabular}

proposed LGC framework for multi-label image classification problems.

\subsection{Sensitivity to Hypothesis Parameters}

The object segmentation hypothesis is critical to the local classifier. While the performance of the object segmentation generator is beyond the scope of this paper, and we could always adopt generator of better performance without touching the structure of the proposed LGC framework, we are still interested in investigating the multi-label classification performance with respect to the number of hypotheses. Figure 3 shows changes in mAP values when we select different number of top hypotheses. The hypotheses are sorted based on the predicted objectness scores [10]. Starting from a small number of hypothesis, object recall rate rise as the number of hypotheses increases, and the mAP score of both local level classification and Local-Global-CNN rise. And the mAP value saturate when top 500 hypotheses are selected (i.e., LGC,82.3\% and Local-

$\mathrm{Cls}, 79.8 \%$ ). When we continue increase the number of hypotheses, the mAP score began to decline. The reason is that more and more noisy hypotheses would influence the accuracy even the recall rate continue rise. 


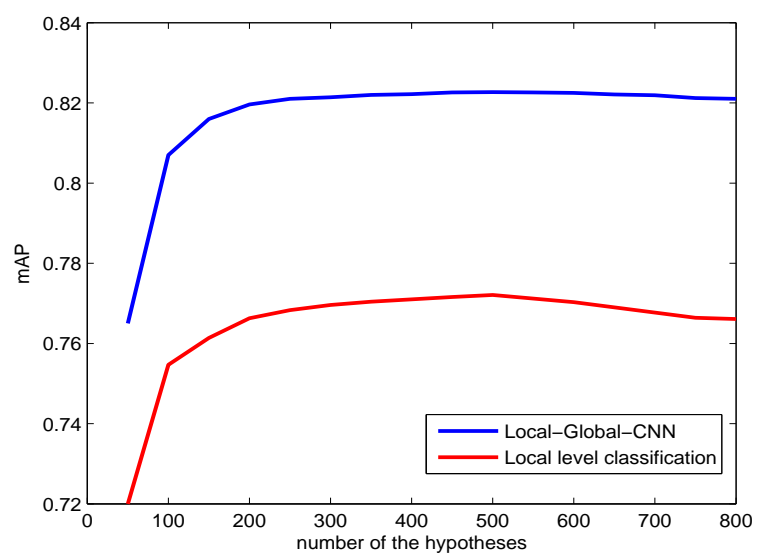

Figure 3: An illustration of changes in mAP values when we select different number of top hypotheses. The hypotheses are sorted based on the predicted objectness scores. At the beginning, as the number of hypotheses increases, mAP values of both local level classification and Local-Global-CNN rise. And the mAP value achieve the highest at selected top 500 hypotheses(i.e.,LGC,82.3\%;Local-Cls,79.8\%.). When we continue increase the number of hypotheses, the mAP values began to decline.

\section{Conclusions}

In this paper we propose a flexible deep CNN infrastructure, called Local-GlobalCNN (LGC). This infrastructure can be divided into local level multi-label classification and global level multi-label classification. Local level multi-label classification pays attention to local region or single object. Global level multi-label classification emphasizes the global level information. In Local level multi-label classification, we take the label co-occurrence information into account to improve classification accuracy by using graphs in the label space. Finally, we integrate predictions of local level multi-label classifier and global level multi-label classifier together as the ultimate multi-label prediction. Experimental results on Pascal VOC2007 and VOC2012 multilabel image dataset show that the proposed method achieves very promising performance in multi-label classification. 
[1] J. Deng, W. Dong, R. Socher, L.-J. Li, K. Li, L. Fei-Fei, Imagenet: A large-scale hierarchical image database, in: Computer Vision and Pattern Recognition, 2009. CVPR 2009. IEEE Conference on, IEEE, 2009, pp. 248-255.

[2] L. Fei-Fei, R. Fergus, P. Perona, Learning generative visual models from few training examples: An incremental bayesian approach tested on 101 object categories, Computer Vision and Image Understanding 106 (1) (2007) 59-70.

[3] G. Griffin, A. Holub, P. Perona, Caltech-256 object category dataset.

[4] D. G. Lowe, Distinctive image features from scale-invariant keypoints, International journal of computer vision 60 (2) (2004) 91-110.

[5] F. Perronnin, J. Sánchez, T. Mensink, Improving the fisher kernel for large-scale image classification, in: Computer Vision-ECCV 2010, Springer, 2010, pp. 143156.

[6] C.-C. Chang, C.-J. Lin, Libsvm: A library for support vector machines, ACM Transactions on Intelligent Systems and Technology (TIST) 2 (3) (2011) 27.

[7] A. Krizhevsky, I. Sutskever, G. E. Hinton, Imagenet classification with deep convolutional neural networks, in: Advances in neural information processing systems, 2012, pp. 1097-1105.

[8] Y. Gong, Y. Jia, T. Leung, A. Toshev, S. Ioffe, Deep convolutional ranking for multilabel image annotation, arXiv preprint arXiv:1312.4894.

[9] J. Xiao, J. Hays, K. Ehinger, A. Oliva, A. Torralba, et al., Sun database: Largescale scene recognition from abbey to zoo, in: Computer vision and pattern recognition (CVPR), 2010 IEEE conference on, IEEE, 2010, pp. 3485-3492.

[10] M.-M. Cheng, Z. Zhang, W.-Y. Lin, P. Torr, Bing: Binarized normed gradients for objectness estimation at 300fps, in: Computer Vision and Pattern Recognition (CVPR), 2014 IEEE Conference on, IEEE, 2014, pp. 3286-3293. 
[11] M. Everingham, L. Van Gool, C. K. Williams, J. Winn, A. Zisserman, The pascal visual object classes (voc) challenge, International journal of computer vision 88 (2) (2010) 303-338.

[12] N. Dalal, B. Triggs, Histograms of oriented gradients for human detection, in: Computer Vision and Pattern Recognition, 2005. CVPR 2005. IEEE Computer Society Conference on, Vol. 1, IEEE, 2005, pp. 886-893.

[13] T. Ojala, M. Pietikäinen, D. Harwood, A comparative study of texture measures with classification based on featured distributions, Pattern recognition 29 (1) (1996) 51-59.

[14] S. Lazebnik, C. Schmid, J. Ponce, Beyond bags of features: Spatial pyramid matching for recognizing natural scene categories, in: Computer Vision and Pattern Recognition, 2006 IEEE Computer Society Conference on, Vol. 2, IEEE, 2006, pp. 2169-2178.

[15] J. Yang, K. Yu, Y. Gong, T. Huang, Linear spatial pyramid matching using sparse coding for image classification, in: Computer Vision and Pattern Recognition, 2009. CVPR 2009. IEEE Conference on, IEEE, 2009, pp. 1794-1801.

[16] J. Wang, J. Yang, K. Yu, F. Lv, T. Huang, Y. Gong, Locality-constrained linear coding for image classification, in: Computer Vision and Pattern Recognition (CVPR), 2010 IEEE Conference on, IEEE, 2010, pp. 3360-3367.

[17] Z. Li, J. Liu, J. Tang, H. Lu, Robust structured subspace learning for data representation, IEEE Transactions on Pattern Analysis and Machine Intelligence 37 (10) (2015) 2085-2098.

[18] J. Tang, Z. Li, M. Wang, R. Zhao, Neighborhood discriminant hashing for largescale image retrieval, IEEE Transactions on Image Processing 24 (9) (2015) $405 \quad 2827-2840$.

[19] Z. Li, J. Liu, Y. Yang, X. Zhou, H. Lu, Clustering-guided sparse structural learning for unsupervised feature selection, IEEE Transactions on Knowledge and Data Engineering 26 (9) (2014) 2138-2150. 
[20] A. Elisseeff, J. Weston, A kernel method for multi-labelled classification, in: Advances in neural information processing systems, 2001, pp. 681-687.

[21] J. Fürnkranz, E. Hüllermeier, E. L. Mencía, K. Brinker, Multilabel classification via calibrated label ranking, Machine learning 73 (2) (2008) 133-153.

[22] M. X. Y.-F. L. Zhi, H. Zhou, Multi-label learning with pro loss.

[23] G. Chen, Y. Song, F. Wang, C. Zhang, Semi-supervised multi-label learning by solving a sylvester equation., in: SDM, SIAM, 2008, pp. 410-419.

[24] F. Kang, R. Jin, R. Sukthankar, Correlated label propagation with application to multi-label learning, in: Computer Vision and Pattern Recognition, 2006 IEEE Computer Society Conference on, Vol. 2, IEEE, 2006, pp. 1719-1726.

[25] H. Wang, H. Huang, C. Ding, Image annotation using multi-label correlated green's function, in: Computer Vision, 2009 IEEE 12th International Conference on, IEEE, 2009, pp. 2029-2034.

[26] Z.-J. Zha, T. Mei, J. Wang, Z. Wang, X.-S. Hua, Graph-based semi-supervised learning with multiple labels, Journal of Visual Communication and Image Representation 20 (2) (2009) 97-103.

[27] X. Li, F. Zhao, Y. Guo, Multi-label image classification with a probabilistic label enhancement model, Proc. Uncertainty in Artificial Intell.

[28] K. Jarrett, K. Kavukcuoglu, M. Ranzato, Y. LeCun, What is the best multi-stage architecture for object recognition?, in: Computer Vision, 2009 IEEE 12th International Conference on, IEEE, 2009, pp. 2146-2153.

[29] B. B. Le Cun, J. S. Denker, D. Henderson, R. E. Howard, W. Hubbard, L. D. Jackel, Handwritten digit recognition with a back-propagation network, in: Advances in neural information processing systems, Citeseer, 1990.

[30] H. Lee, R. Grosse, R. Ranganath, A. Y. Ng, Convolutional deep belief networks for scalable unsupervised learning of hierarchical representations, in: Proceed- 
ings of the 26th Annual International Conference on Machine Learning, ACM, 2009, pp. 609-616.

[31] A. Krizhevsky, G. Hinton, Learning multiple layers of features from tiny images (2009).

[32] J. Weston, S. Bengio, N. Usunier, Wsabie: Scaling up to large vocabulary image annotation, in: IJCAI, Vol. 11, 2011, pp. 2764-2770.

[33] Y. Wei, W. Xia, J. Huang, B. Ni, J. Dong, Y. Zhao, S. Yan, Cnn: Single-label to multi-label, arXiv preprint arXiv:1406.5726.

[34] J. Donahue, Y. Jia, O. Vinyals, J. Hoffman, N. Zhang, E. Tzeng, T. Darrell, Decaf: A deep convolutional activation feature for generic visual recognition, arXiv preprint arXiv:1310.1531.

[35] R. Girshick, J. Donahue, T. Darrell, J. Malik, Rich feature hierarchies for accurate object detection and semantic segmentation, in: Computer Vision and Pattern Recognition (CVPR), 2014 IEEE Conference on, IEEE, 2014, pp. 580-587.

[36] A. S. Razavian, H. Azizpour, J. Sullivan, S. Carlsson, Cnn features off-the-shelf: an astounding baseline for recognition, in: Computer Vision and Pattern Recognition Workshops (CVPRW), 2014 IEEE Conference on, IEEE, 2014, pp. 512-519.

[37] B. Alexe, T. Deselaers, V. Ferrari, Measuring the objectness of image windows, Pattern Analysis and Machine Intelligence, IEEE Transactions on 34 (11) (2012) 2189-2202.

[38] J. Carreira, C. Sminchisescu, Cpmc: Automatic object segmentation using constrained parametric min-cuts, Pattern Analysis and Machine Intelligence, IEEE Transactions on 34 (7) (2012) 1312-1328.

[39] M.-M. Cheng, J. Warrell, W.-Y. Lin, S. Zheng, V. Vineet, N. Crook, Efficient salient region detection with soft image abstraction, in: Computer Vision (ICCV), 2013 IEEE International Conference on, IEEE, 2013, pp. 1529-1536. 
[40] J. R. Uijlings, K. E. van de Sande, T. Gevers, A. W. Smeulders, Selective search for object recognition, International journal of computer vision 104 (2) (2013) 154-171.

[41] H. Harzallah, F. Jurie, C. Schmid, Combining efficient object localization and image classification, in: Computer Vision, 2009 IEEE 12th International Conference on, IEEE, 2009, pp. 237-244.

[42] P. Sermanet, D. Eigen, X. Zhang, M. Mathieu, R. Fergus, Y. LeCun, Overfeat: Integrated recognition, localization and detection using convolutional networks, arXiv preprint arXiv:1312.6229.

[43] M. Oquab, L. Bottou, I. Laptev, J. Sivic, Learning and transferring mid-level image representations using convolutional neural networks, in: Computer Vision and Pattern Recognition (CVPR), 2014 IEEE Conference on, IEEE, 2014, pp. $1717-1724$.

[44] Y. LeCun, M. Ranzato, Deep learning tutorial, in: Tutorials in International Conference on Machine Learning (ICML13), Citeseer, 2013. 